\title{
Prädiktoren des Blutungsrisikos
}

Fragestellung: Lässt sich bei Patienten mit zufällig entdeckten zerebralen Kavernomen das Blutungsrisiko voraussagen?

Hintergrund: Kavernome sind in vielen Fällen Zufallsentdeckungen, wenn Kernspintomografien des Gehirns wegen anderen Indikationen durchgeführt werden. Die Prävalenz bei neurologisch unauffälligen Personen liegt zwischen 1:600 und 1:700. Kavernome können symptomatisch werden, indem sie bluten oder zu epileptischen Anfällen führen. Selten bedingen sie andere fokal-neurologische Ausfälle. Die gefürchtetste Komplikation von Kavernomen ist die Blutung. Die meisten Daten zum Blutungsrisiko beruhen bisher auf retrospektiven Auswertungen. Die vorliegende Publikation ist die erste größere Studie, die auf Daten individueller Patienten beruht.

Patienten und Methodik: Die Autoren identifizierten StudienZentren, die Kohorten von Patienten mit Kavernomen im Alter über 16 Jahren publiziert hatten. Die Zentren wurden angeschrieben und gebeten, die Base-

Horne MA, Flemming KD, Su IC et al. Clinical course of untreated cerebral cavernous malformations: a meta-analysis of individual patient data. Lancet Neurol 2016; 15: 166-73 line-Daten der Patienten und Ereignisse während der weiteren Beobachtung zu erfassen. Aus den so gewonnenen Daten wurde dann das 5-JahresRisiko einer symptomatischen intrakraniellen Blutung mithilfe einer multivariablen Cox-Regressionsanalyse berechnet, um Prädiktoren für eine Blutung zu erhalten.

Ergebnisse: Die Studie umfasste 1.620 Personen aus sieben Kohorten. Während 5.197 Personenjahren traten 204 intrakranielle Blutungen auf. Das entspricht einem 5-Jahres-Risiko von 15,8\% (95\%-Konfidenzintervall [KI] 13,7-17,9\%). Das Risiko war deutlich erhöht, wenn ein Kavernom bereits geblutet hatte oder symptomatisch war. Bei im Hirnstamm lokalisierten Kavernomen war das Blutungsrisiko um den Faktor 4,4 erhöht gegenüber Kavernomen in anderen Lokalisationen. Keinen Einfluss auf das Blutungsrisiko hatten Alter, Geschlecht und die Zahl der Kavernome. Das 5-Jahres-Risiko einer intrazerebralen Blutung bei unbehandelten Patienten betrug 3,8\% für Personen mit Kavernomen außerhalb des Hirnstamms ohne Symptome, $8 \%$ bei Kavernomen im Hirnstamm ohne Blutung und Symptome, $18,4 \%$ für Personen mit Kavernomen außerhalb des Hirnstamms mit Blutung oder fokal-neurologischen Defiziten und 30,8\% für Personen mit Kavernomen im Bereich des Hirnstamms, die bereits geblutet hatten oder fokal-neurologische Defizite aufwiesen.

Schlussfolgerungen: Die bisher größte Kohortenstudie zur Prognose von unbehandelten zerebralen Kavernomen zeigte, dass Patienten mit Kavernomen im Hirnstamm, die bereits geblutet haben oder fokal-neurologische Defizite verursachen, das höchste Blutungsrisiko aufweisen.

\section{- Kommentar von Hans-Christoph Diener, Essen}

\section{Basis für eine randomisierte Therapiestudie}

Diese Publikation ist die numerisch größte untersuchte Population zur Prognose von zerebralen Kavernomen. Die Studie zeigte ein relativ geringes Blutungsrisiko, wenn Kavernome außerhalb des Hirnstamms lokalisiert sind, bisher nicht geblutet haben und keine fokal-neurologischen Defizite verursachen. Hier besteht sehr wahrscheinlich kein unmittelbarer Handlungsbedarf. Kavernome im Hirnstamm, insbesondere diejenigen, die bereits geblutet haben, haben ein sehr hohes Blutungsrisiko und in diesen Fällen muss mit den Neurochirurgen kritisch diskutiert werden, ob eine operative Resektion des Kavernoms indiziert und möglich ist.

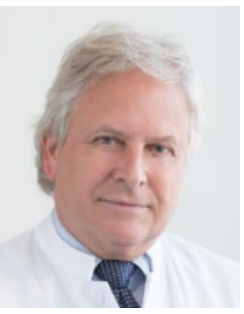

Prof. Dr. med. Hans-Christoph Diener, Essen

Direktor der Klinik für Neurologie, Universitätsklinikum Essen

E-Mail:h.diener@uni-essen.de
Eine weitere Indikation für die Operation eines Kavernoms, das nicht im Hirnstamm gelegen ist, stellt die Vermutung dar, dass das Kavernom als Quelle für fokal-eingeleitete und sekundär-generalisierte Anfälle infrage kommt, wenn diese medikamentös nicht zu beherrschen sind. Die hier festgestellten Ereignisraten und Risikofaktoren bilden eine gute Grundlage, um ihn Zukunft eine randomisierte Therapiestudie zu planen.

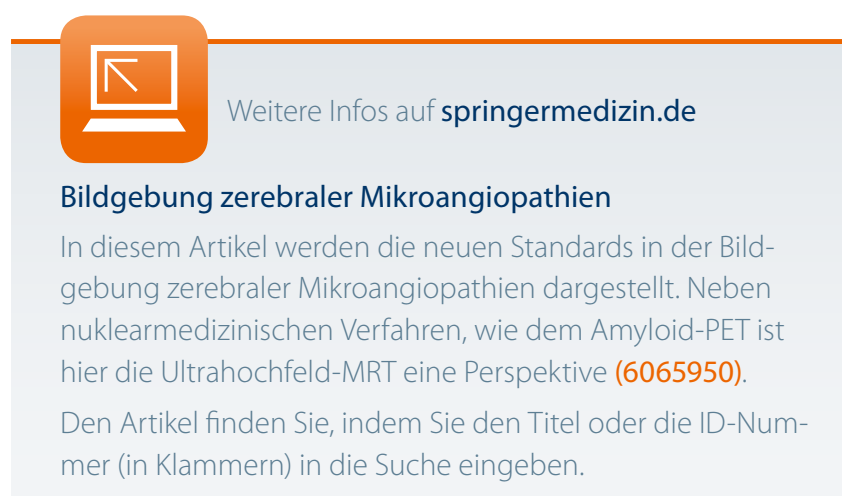

